\title{
Microgels at Interfaces Behave as 2D Elastic Particles Featuring Reentrant Dynamics
}

\author{
Fabrizio Camerin $\odot,{ }^{1,2, *}$ Nicoletta Gnan $\odot,{ }^{1,3}$ José Ruiz-Franco $\odot,{ }^{3,1}$ Andrea Ninarello®, ${ }^{1,3}$ \\ Lorenzo Rovigatti®, ${ }^{3,1}$ and Emanuela Zaccarelli॰ $\oplus^{1,3, \uparrow}$ \\ ${ }^{1}$ CNR Institute of Complex Systems, Uos Sapienza, Piazzale Aldo Moro 2, 00185 Roma, Italy \\ ${ }^{2}$ Department of Basic and Applied Sciences for Engineering, Sapienza University of Rome, \\ via Antonio Scarpa 14, 00161 Roma, Italy \\ ${ }^{3}$ Department of Physics, Sapienza University of Rome, Piazzale Aldo Moro 2, 00185 Roma, Italy
}

(Received 15 January 2020; revised 9 April 2020; accepted 15 May 2020; published 15 July 2020)

Soft colloids are increasingly used as model systems to address fundamental issues such as crystallization and the glass and jamming transitions. Among the available classes of soft colloids, microgels are emerging as the gold standard. Since their great internal complexity makes their theoretical characterization very hard, microgels are commonly modeled, at least in the small-deformation regime, within the simple framework of linear elasticity theory. Here we show that there exist conditions where its range of validity can be greatly extended, providing strong numerical evidence that microgels adsorbed at an interface follow the two-dimensional Hertzian theory, and hence behave like 2D elastic particles, up to very large deformations, in stark contrast to what found in bulk conditions. We are also able to estimate Young's modulus of the individual particles and, by comparing it with its counterpart in bulk conditions, we demonstrate a significant stiffening of the polymer network at the interface. Finally, by analyzing dynamical properties, we predict multiple reentrant phenomena: By a continuous increase of particle density, microgels first arrest and then refluidify due to the high penetrability of their extended coronas. We observe this anomalous behavior in a range of experimentally accessible conditions for small and loosely cross-linked microgels. The present work thus establishes microgels at interfaces as a new model system for fundamental investigations, paving the way for the experimental synthesis and research on unique highdensity liquidlike states. In addition, these results can guide the development of novel assembly and patterning strategies on surfaces and the design of novel materials with desired interfacial behavior.

DOI: $10.1103 /$ PhysRevX.10.031012

\section{INTRODUCTION}

Mesoscopic assemblies of colloids and nanoparticles display features that depend critically on the microscopic details of the building blocks, e.g., composition, size, and shape, as well as on the specific macroscopic physical conditions such as the thermodynamic control parameters. It is by carefully choosing and tuning these variables that one can induce the formation of different structures and explore various states, such as liquidlike fluids, glasses, or crystals [1]. At the core of this collective behavior is the interparticle interaction, which ultimately dictates the phase

\footnotetext{
* Corresponding author. fabrizio.camerin@uniroma1.it

Corresponding author. emanuela.zaccarelli@cnr.it

Published by the American Physical Society under the terms of the Creative Commons Attribution 4.0 International license. Further distribution of this work must maintain attribution to the author(s) and the published article's title, journal citation, and DOI.
}

Subject Areas: Materials Science, Soft Matter, Statistical Physics behavior and dynamics of the assembly: Information at the multiparticle level is thus fundamental to determine the properties of the material. If a system is made of rigid building blocks that interact only through excluded volume interactions, it can be approximately mapped to a hardsphere system, and its behavior can be investigated through packing models. These approaches have been used for a long time to successfully answer fundamental questions in physics and material science whenever simple constituent units are involved [2].

However, in certain cases, the complexity that resides at the microscopic level cannot be described in these terms. This is especially true for soft polymeric colloids that possess internal degrees of freedom endowing them with elasticity and deformability. Among the available library of soft deformable particles, microgels, colloidal-sized crosslinked polymer networks, are one of the finest illustrations of this concept [3-7]. Their structure is determined by the chemical synthesis conditions that, in the common procedure of precipitation polymerization [8], lead to the formation of spherical particles made of a compact core and a fluffy external corona [9]. Although microgels are 
often considered as simple elastic particles that can be modeled with the classical elasticity theory, the presence of multiple length scales in their internal architecture makes their effective interactions in bulk more complex than a pure Hertzian model and calls for more refined treatments that range from a phenomenological multi-Hertzian model [10] to descriptions that depend on the concentration regime [11,12].

The intrinsic softness of microgels and, in general, of soft deformable objects, is fully revealed, and can be taken advantage of, at interfaces, which can be used to fulfill different purposes. In fact, if the interfacial tension is large enough, it is possible to coat an interface with nano- or microsized particles which then remains adsorbed and can form stable monolayers. This concept can be used, for instance, to stabilize emulsions and biological membranes [13-17] or to study fundamental self-assembly phenomena in 2D such as crystal [18] or quasicrystal formation [19,20]. Moreover, by changing in situ the single-particle properties and the local environment, it is possible, in principle, to finely control the stability and the structure of the whole monolayer or emulsion [21-24]. Noteworthy, in this respect, is the possibility to realize complex patterns whose application as etching masks in nanolithography can lead, for instance, to the fabrication of nanowire arrays [25,26].

When adsorbed at interfaces, microgels flatten out and adopt the so-called "fried-egg" shape, making them very different from their bulk counterparts [27-29]. In particular, at an interface, the polymer network tends to minimize as much as possible the surface tension between the two liquids by taking a stretched configuration already in the dilute regime. So far, the effects that an interfacial confinement induces on such particles have been mainly limited to the study of their characteristic shape, and hence, to their structural arrangement at oil-water and air-water interfaces. These aspects have been widely investigated experimentally $[30,31]$ and, more recently, also numerically $[29,32,33]$.

By contrast, little is known about the collective behavior of such particles, mostly by means of indirect experimental feedback [34-38], which has neither allowed us to extract a functional form for the interaction potential nor to properly understand the role of the surface tension. In the same way, a true characterization of the elasticity of the polymer network within the interfacial plane is still missing, being limited both experimentally and numerically by subpar techniques and models. Clearly, a simple transfer of results from bulk to interface would be highly inappropriate, due to the dramatic change of conditions between the two cases.

This lack of understanding hampers the progress toward further applications, since an established fundamental knowledge of the basic constituents would make it possible to a priori design and guide the assembly of innovative materials and nanostructures. From a theoretical standpoint, it also prevents the adoption of microgels at interfaces as model systems for the study of open questions in fundamental science $[39,40]$. In this sense, it is crucial to provide a microscopic understanding of such a system.

In this work, we address this problem by calculating both the effective interactions between two microgels at a liquidliquid interface and their individual elastic properties, using this knowledge to predict their multiparticle response at high densities. Our approach relies on state-of-the-art modeling of single-particle microgels that was shown to quantitatively capture the internal topology of laboratory microgels both in the bulk [41] and at the interface [29]. In the latter case, an appropriate framework, that explicitly takes into account the effects of the surface tension between the two liquids, has been developed in order to correctly describe the deformation of the microgels [29]. Despite the complex arrangement of the polymer network at the interface and the intrinsic presence of a core-corona structure, the calculation of the effective interactions between two microgels on the interfacial plane reveals a remarkable agreement with the $2 \mathrm{D}$ expression of the Hertzian model for elastic disks for all investigated distances and cross-linker concentrations $c$. This is clearly different from what was found for the same system in bulk conditions [42] and establishes the validity of the twodimensional Hertzian model for microgels at interfaces up to large compression regimes. Young's modulus determined from the effective potential is also directly compared to explicit calculations based on elasticity theory for small and intermediate deformations. Thanks to this method, we are able to achieve a full characterization of the elastic response of the microgels in the two-dimensional interfacial plane, and we can thus establish a sound comparison to the three-dimensional bulk case. Notably, our results show that the elastic moduli, once converted to their threedimensional counterparts, are roughly one order of magnitude larger at the interface than for the same microgels in the bulk. This highlights the key role of the interfacial tension in stiffening the microgels due to the stretching of their coronas.

Having determined how such complex particles interact with each other, we are finally able to carry out our study also at the collective level by investigating the dynamical phase behavior of an ensemble of these effective elastic disks. When softness and elasticity are taken into account in the interparticle interaction, a rich behavior is, in general, expected [43-45]. In particular, we find the presence of multiple reentrant melting phenomena, where a glass is melted simply by an increase in particle concentration. Although similar findings have long been predicted for simple soft models [46], here, for the first time, such a scenario is found for microscopically motivated effective interactions and, most importantly, for potential parameters that can be realized in experiments.

The extensive analysis of microgels at the interface presented here, together with the notion that the Hertzian model can be used up to very large deformation energies, sheds light on how single microgel properties and 
collective response are coupled, and demonstrates that this system is an extremely promising model for probing the collective behavior of 2D elastic particles up to large densities. Their unusual dynamical features are of wide interest for the preparation of colloidal monolayers with nonmonotonic viscoelastic properties that could be used for a variety of different applications.

\section{THE MODEL}

Microgel monomers interact through the Kremer-Grest bead-spring model $[47,48]$ in explicit solvent. The polymer network has a disordered topology [49] and the distribution of cross-linker is inhomogeneous, slowly rarefying toward the corona, as typically results from microgels synthesized via precipitation polymerization. These factors allow for a favorable comparison to realistic microgels, both in terms of form factors and density profiles [41]. The solvent is treated within the dissipative particle dynamics (DPD) framework [50] and, for simulations at the interface, their mutual interactions are tuned to mimic a water-hexane interface. The surface tension between the two fluids is representative of a wide variety of solvents that are typically employed for such studies at the interface. Furthermore, no relevant differences in the distribution of the microgel

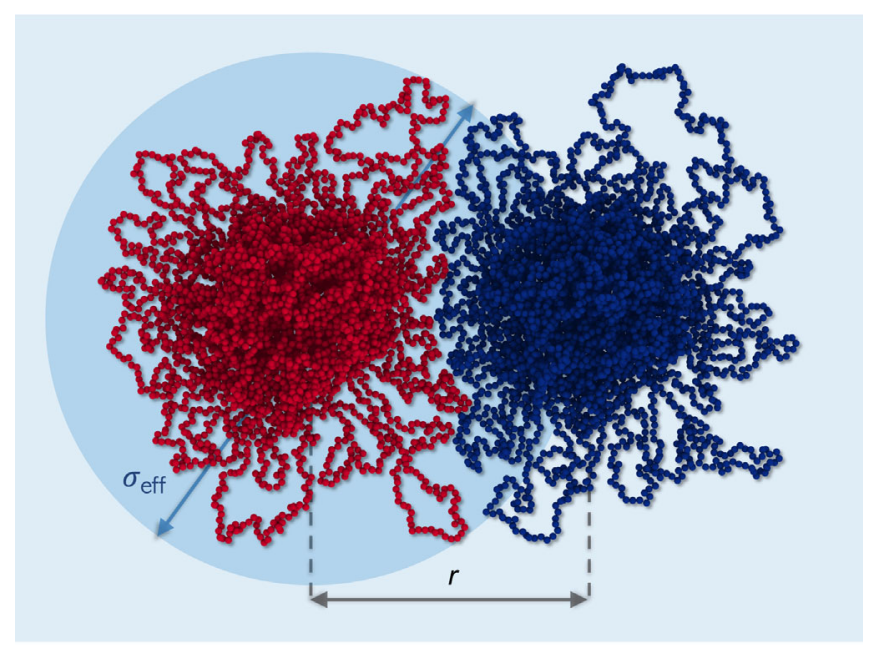

Oil

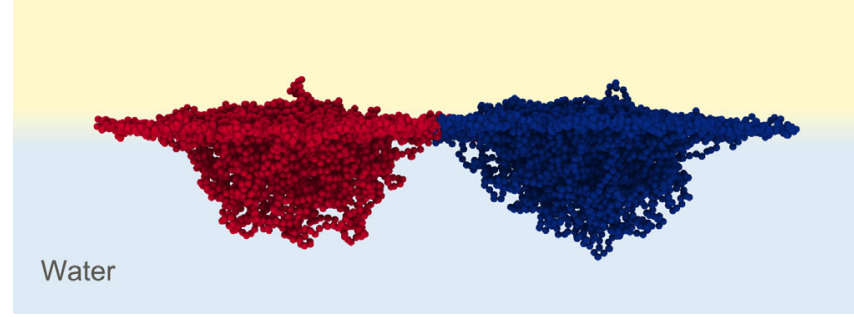

FIG. 1. Microgels interacting at the interface. Top and side simulation snapshots of two microgels with $c=5 \%$ at the wateroil interface at a representative distance $r \approx 40 \sigma_{m}$. The effective diameter of the microgel is $\sigma_{\text {eff }}$. Solvent particles are not shown for clarity. monomers on the plane of the interface are expected by changing the value of the surface tension [29].

Under these conditions, the microgel spontaneously adopts the characteristic "fried-egg" shape when placed close to the interfacial plane. Its structural characterization is in good agreement with experiments both in terms of flattening on the interfacial plane and of protrusion on the preferred water side [29]. In the present work, for the study of the microgel-microgel effective interactions, we simulate particles with $N \approx 5000$ monomers of diameter $\sigma_{m}$, that defines the unit of length, and cross-linker molar fraction $c=3 \%, 5 \%$, and $10 \%$. Because of the exceptional computational cost to carry out the simulations, we limit our study to a single microgel topology for each studied value of $c$, checking the consistency of the results with a second topology for $c=5 \%$ (see Supplemental Material [51]). The elastic properties of smaller microgels with 2000 and 3000 monomers are also analyzed for further considerations on their collective behavior. Additional details on the microgel and interface modeling, and on simulations are provided in Sec. V and in Refs. [29,41,50]. The typical conformation taken by two interacting microgels at the interface is reported in the simulation snapshots of Fig. 1.

\section{RESULTS AND DISCUSSION}

\section{A. Effective interaction potential}

The two-body effective potential $V_{\text {eff }}(r)$ between the microgels at a water-hexane interface is evaluated by means of extensive simulations exploiting the umbrella sampling technique [52-54], as also explained in Sec. V, and it is shown in Fig. 2(a) for all investigated values of $c$, rescaled by $\beta=1 / k_{B} T$. The numerical results are compared to the two-dimensional Hertzian expression $[55,56]$ that reads as

$$
V_{H}(r)=\frac{\frac{1}{2} \pi Y \sigma_{\mathrm{eff}}^{2}\left(1-\frac{r}{\sigma_{\mathrm{eff}}}\right)^{2}}{\ln \left(\frac{2}{1-\frac{r}{\sigma_{\mathrm{eff}}}}\right)},
$$

where $r$ is the distance between the centers of mass of the microgels at the interface, $\sigma_{\text {eff }}$ quantifies the effective microgel diameter on the interfacial plane, and $Y$ is Young's modulus of the individual particle. The agreement between the numerical results and the theoretical fits is remarkable for all probed distances and all studied values of $c$. Therefore, it clearly emerges from these findings that two microgels confined at an interface effectively behave as 2D elastic objects, further confirming the soft repulsive nature of their mutual interactions. Further discussion on the functional form of the potential can be found in the Supplemental Material [51].

Experimentally, small microgels-having a diameter $\lesssim 200 \mathrm{~nm}$-are the best candidates to interact in this way, since they do not experience long-range attractions due to capillary effects [37]. Indeed, the latter conditions 


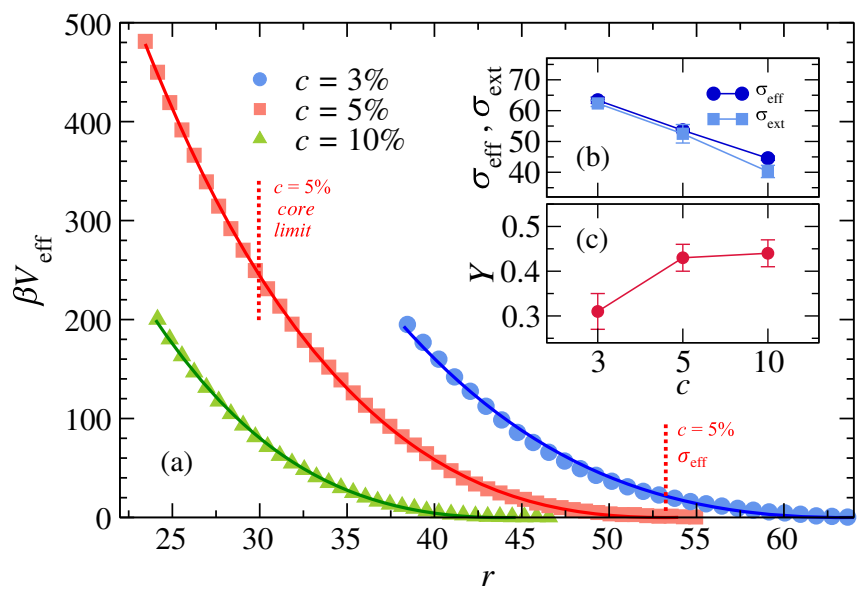

FIG. 2. Effective potentials for microgels at the liquid-liquid interface and related 2D Hertzian fit parameters. (a) Numerical results refer to three values of the cross-linker concentrations $c$ : $3 \%$ (circles), 5\% (squares), and 10\% (triangles). Full lines are fits to numerical results using Eq. (1). The two vertical dotted lines indicate the value $\sigma_{\text {eff }} \approx 53 \sigma_{m}$ and the distance at which the cores of the two microgels get in contact $\left(r \approx 30 \sigma_{m}\right)$ for $c=5 \%$; (b) Microgel effective diameter $\sigma_{\text {eff }}$ compared to the extension on the plane of the interface $\sigma_{\text {ext }}$ calculated as in Ref. [29], in units of $\sigma_{m}$; (c) Young's modulus $Y$ extracted from the 2D Hertzian fit to $V_{H}$ in units of $k_{B} T / \sigma_{m}^{2}$.

have been widely reported [57-59] and found to be relevant only for microgels large enough to induce a local deformation of the water-hexane interface [37]. By contrast, our solvent modeling is aimed essentially at reproducing the surface tension and the microgel solubility, both of which have a direct influence on the conformation of the particle. We can thus directly probe the elastic interactions between the microgels without the interference of attractive capillary forces.

These outcomes also evidence the presence of a single characteristic length in the potential up to a center-to-center distance as small as the interaction radius of the microgel (approximately $\sigma_{\text {eff }} / 2$ ) for the case $c=5 \%$, which we probe up to a repulsion of approximately $500 k_{B} T$. The observed behavior is strikingly different from the corresponding bulk one, where the Hertzian potential is found to be valid only up to a few $k_{B} T$ 's [42]. Indeed, in bulk, the distinction between core and corona imposes us to consider different kinds of interactions, depending on the investigated distances [10], that would describe different inner regions of the particle with changing elastic properties. Instead, at an interface, the microgel behaves as if the polymer network were more homogeneous and uniform, as indicated by the continuous and steady growth of the potential that persists even inside the core region, here corresponding to $r \lesssim 30 \sigma_{m}$ for $c=5 \%$, as also reported in Fig. 2(a). This behavior suggests a dominant role of the surface tension which completely controls the properties of the microgel at the interface, so that even the part of the core that protrudes from the plane of the interface effectively contributes to the 2D Hertzian description. Thus, microgels adsorbed at interfaces represent the first colloidal system to behave as an ideal Hertzian model, when considered as two-dimensional objects on the interfacial plane. Their behavior is thus clearly different from that of microgels in bulk [10,42].

By fitting the calculated potential with Eq. (1), we can obtain the effective diameter $\sigma_{\text {eff }}$ of the flattened microgel and its Young's modulus $Y$. Interestingly, the latter quantity can be also directly estimated from the fit of the calculated potentials, at odds with the corresponding 3D case where two nonindependent elastic parameters, namely, $Y$ and Poisson's ratio $\nu$, are contained in the Hertzian prefactor [42]. The resulting fit parameters are shown in Figs. 2(b) and 2(c). In particular, the effective diameter is found to be very close, at all $c$, to the microgel extension $\sigma_{\text {ext }}$, displayed in Fig. 2(b), that can be estimated by taking opposite edges of the microgel on the interfacial plane [29]. The slight underestimation of $\sigma_{\text {ext }}$ as compared to $\sigma_{\text {eff }}$ is associated with the fact that effective interaction calculations are also sensitive to the outer dangling chains. This information is partially lost by averaging over the distance of all opposite sites on the plane of the interface. As expected, the extension of the microgel at the interface decreases as a function of $c$ in agreement with experiments [29], since softer microgels deform more strongly, and hence spread more at the interface. The corresponding values of $Y$ are reported in Fig. 2(c), showing that higher cross-linking leads to stiffer networks, following expectations and in agreement with findings for microgels in bulk [42].

\section{B. Elasticity theory calculations}

The estimate of Young's modulus extracted from the fit can be compared to the one obtained through the use of elasticity theory in 2D. In this framework, one can evaluate the area and shape fluctuations of the microgel on the plane of the interface, writing the elastic energy $U$ as a function of the two strain invariants of the strain tensor $[60,61]$. In this case, we write $U$ according to the phenomenological Mooney-Rivlin theory, that is known to be valid also beyond the linear elastic regime. Within this theoretical approach, we can calculate all the elastic moduli of a microgel from equilibrium simulations, as previously done for microgels in bulk [42]. In particular, the moduli refer to the two-dimensional projection of the microgel on the interface, assuming that they are dominated by corona fluctuations. In order to compare with the corresponding bulk properties, we also perform a similar procedure in 3D for the same microgel topologies in the presence of explicit solvent (see Ref. [42] for the implicit solvent treatment). However, bulk and interfacial moduli are naturally given in different units, so we adopt the so-called plane-stress approximation for the $2 \mathrm{D}$ moduli [62]. In this way, we assume that the stress normal to the interface is zero $[63,64]$, a legitimate assumption for two-dimensional objects. Hence, 


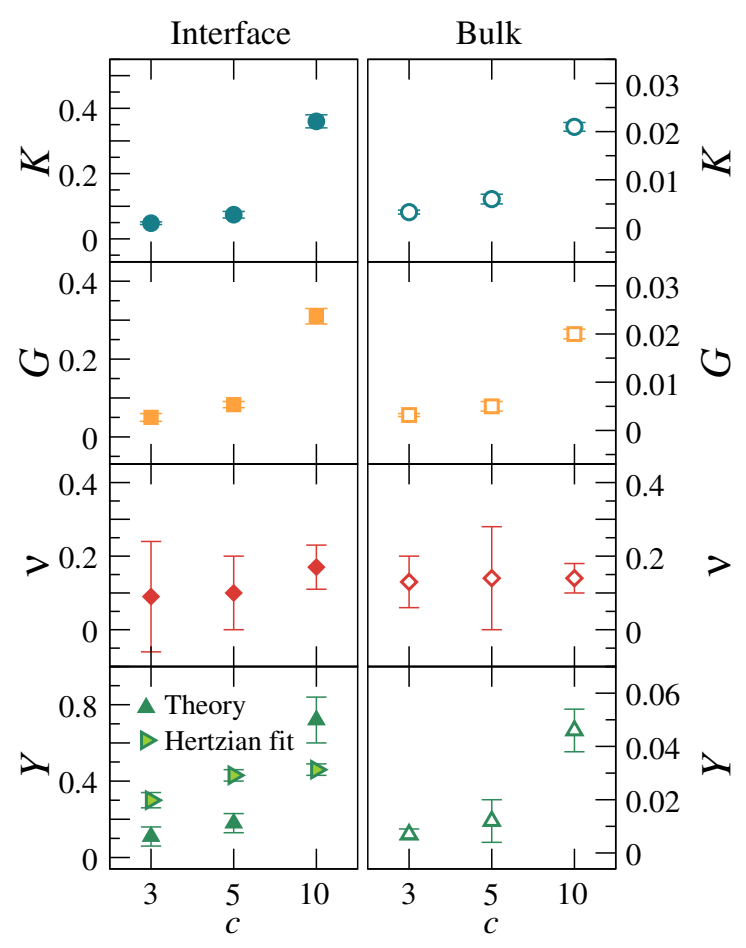

FIG. 3. Elastic moduli at the interface and in the bulk. Bulk modulus $K$, shear modulus $G$, Poisson's ratio $\nu$, and Young's modulus $Y$ for the same microgel topology at the interface (full symbols) and in bulk (empty symbols) with explicit solvent as a function of $c$. In the last row, the theoretical results for $Y$ are also compared to the ones obtained from the effective potential fits with the Hertzian model [Eq. (1)], also reported in Fig. 2(c). $K, G$, and $Y$ are in units of $k_{B} T / \sigma_{m}^{3}$ to appropriately compare bulk and interface moduli, where the latter moduli are divided by the thickness of the shell at the interface (approximately $\sigma_{m}$ ); $\nu$ is dimensionless. Error bars estimated from the fits of $P(J)$ for $K$ and $P(I)$ for $G$ (see Sec. V) are propagated in the calculation of $\nu$ and $Y$.

we consider the small thickness of the microgels normal to the interface to be roughly comparable to the monomer size (approximately $\sigma_{m}$ ), and divide the obtained 2D moduli by this length. We can finally convert them into the corresponding 3D moduli for very thin three-dimensional objects using the relations reported by Torquato [62] for plane-stress conditions. More details on these calculations are provided in Sec. V and in the Supplemental Material [51].

The resulting elastic moduli are reported in Fig. 3 as a function of the cross-linker concentration both for microgels at interfaces (left panels) and in bulk (right panels). Overall, we observe a monotonic increase of $K, G$, and $Y$ as a function of $c$, while $\nu$ remains nearly constant. We note that the nonlinear dependence of $G$ and $Y$ on $c$ is to be expected, since the chains are not Gaussian and the network contains both dangling ends and loops $[65,66]$. These trends are preserved both in the bulk and at the interface. We stress that our two independent estimates of Young's modulus, namely, the one provided by the Mooney-Rivlin theory and that obtained by the 2D Hertzian fitting, also reported in Fig. 3, are consistent with each other. We highlight in this way how the single-particle properties of a microgel at an interface are fully reflected in the multiparticle behavior. The most striking result of this analysis is the fact that all three moduli at the interface are significantly larger, by approximately one order of magnitude, than their respective bulk counterparts. As for Poisson's ratio, even though we find similar values in both cases, it should be noted that its upper limit in $2 \mathrm{D}$ is 1.0 while in $3 \mathrm{D}$ it is 0.5 [67].

These findings provide robust evidence of the reduced flexibility of the microgels at a liquid-liquid interface, an issue that up to now has either been extracted from indirect results or sometimes related to charge effects $[68,69]$. Instead, we directly prove that it is entirely attributable to the presence of the interface, where microgels assume a much more stretched configuration with respect to their standard arrangement in bulk. We are able to establish this link thanks to the relative simplicity of our model, whereby a neutral microgel spontaneously adsorbs at the interface without any externally imposed confinement. Under these conditions, microgels are much more resistant to deformation. Indeed, the corona is completely extended and restrained at the interface with the polymer chains being much less responsive to external forces than in bulk, while still minimizing the surface tension. We further note that no available experimental results have so far reported the lateral elastic response of the microgels on the interfacial plane but rather the perpendicular one over a solid substrate [70]. The lateral response is supposed to be the relevant one for the formation of thin microgel layers or for pattern formation on surfaces [25].

\section{Multiparticle dynamical response}

The level of coarse graining adopted up to now has allowed us to describe how the properties of single constituents affect their mutual interactions. Now we go one step further by investigating the multiparticle behavior, i.e., the condition where many microgel particles interact on the interfacial plane. To shed light on this aspect, we simulate a system of particles whose interaction potential is the one we extracted previously, that is the 2D Hertzian potential. In this way, by further coarse graining our system, we are able to assess, for instance, the dynamical response of microgels that are adsorbed on the interfacial plane.

The research on the phase behavior of soft colloids has recently gained much interest: Being the archetype potential to describe interactions among elastic particles, the Hertzian phase diagram has been studied both in three $[46,71]$ and in two dimensions $[72,73]$. In the latter case, however, the investigations that have been carried out were limited to a change in the value of the exponent of the well-known 3D Hertzian without considering that a variation in the dimensionality of the problem implies a 
change in the functional form itself. Indeed, the logarithmic correction arising in Eq. (1) cannot be properly captured by a simple variation in the Hertzian exponent.

We perform Langevin dynamics simulations of 2D Hertzian particles for different area fractions $\phi$ and varying the strength of the 2D Hertzian $A=\pi Y \sigma_{\text {eff }}^{2} /(2 \ln 2)$, which corresponds to the $r \rightarrow 0$ limit in Eq. (1). In order to have access to the dynamical response, we avoid crystallization by introducing polydispersity in the system (see Sec. V). It is important to notice that, in our simulations, particles are assumed to have fixed size, different from bulk conditions where recent simulations and experiments have shown that deswelling plays an important role for concentrated microgel suspensions [74-76]. Instead, there is no reported evidence of deswelling when microgels are compressed at the interface. This is again due to the dominant role of the surface tension which makes adsorbed microgels much less responsive to external stimuli $[32,35]$. In this way, their compression is simply associated with a smooth and monotonic decrease of their interparticle distance, as described experimentally in Ref. [37].

Figure 4(a) reports the self-diffusion coefficients $D$ extracted from the long-time behavior of the mean-squared displacements of the effective microgels for different values of $\phi$ and $A$. We consider the system to fall out of equilibrium on the simulation timescale when $D$ decreases by roughly three orders of magnitude with respect to its low-density value. Thus, we assume the system to be arrested for $D \lesssim 2 \times 10^{-5}$ (in simulation units).

Importantly, we reveal the onset of two clear reentrant melting phenomena where the diffusivity, at first, decreases leading to the formation of a glassy system and then it grows again. This increase takes place primarily for $\phi \gtrsim 1.5$ with a local maximum emerging at $\phi \sim 1.9$. For higher densities, after a further slowdown, the system refluidifies again acquiring a finite diffusion coefficient. Interestingly, at the new local maximum appearing for $\phi \sim 2.5$, the value of $D$ is even larger than that at the previous maximum.

Previous works have shown that one can estimate the locus of the glass transition by monitoring the so-called isodiffusivity (iso- $D$ ) lines [77-79], along which $D$ remains constant. Importantly, it has been shown that the iso- $D$ lines always maintain, for not too large values of the probed $D$, the same shape as the ultimate line of arrest. Thus, by extrapolating to the $D \rightarrow 0$ limit, it is possible to locate the glassy region of a system. By taking a set of different isodiffusivity lines in Fig. 4(a), we draw the corresponding fluid-glass state diagram for the 2D Hertzian model shown in Fig. 4(b). We notice that for the present system, a fluidlike region persists at high densities for $\beta A \lesssim 1100$. We also stress that similar reentrant features in the dynamics have long been predicted in the three-dimensional version of the Hertzian potential [46] and in extensive simulations of monomer-resolved single-chain nanoparticles [80]. This phenomenon is typically linked to the soft nature of the interaction potential that, in contrast to hard-core ones where the packing of the particles is limited by excluded volume interactions, make it possible to restore long-time diffusive motion at high densities, thanks to a balance between energetic and entropic contributions, as also observed in simulations of the Gaussian core model [81] or of the star polymer potential [78]. Nevertheless, reentrant transitions have never been found in experiments of soft [82] and ultrasoft colloids [83]. While microgels in bulk conditions do not show high-density liquid states due to their deviations from an ideal Hertzian behavior [10,11], as also confirmed experimentally $[12,82,84]$, those at interfaces stand as optimal candidates for displaying such an intriguing dynamical behavior.

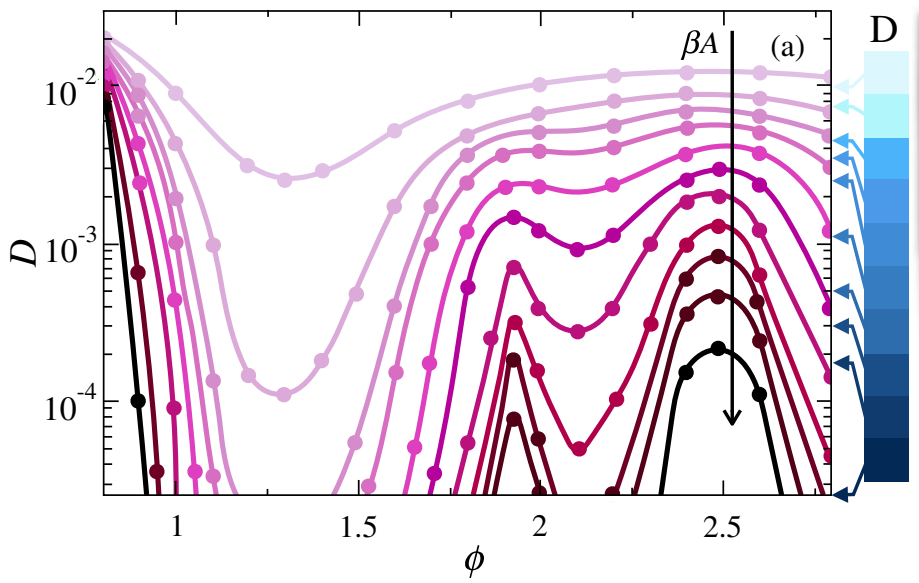

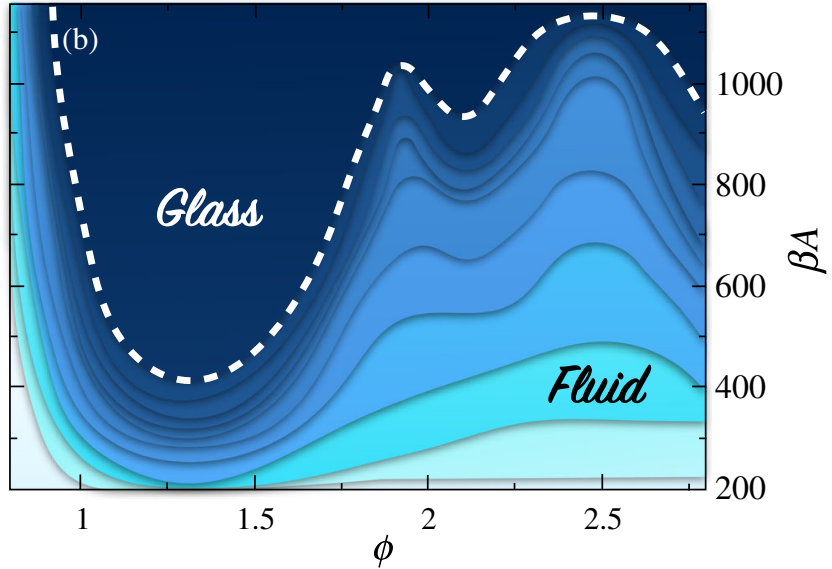

FIG. 4. 2D Hertzian phase diagram. (a) Diffusion coefficient $D$ as a function of the area fraction $\phi$ for different values of the 2D Hertzian strength $A$. From top to bottom, $A$ takes the following values: 226, 340, 409, 453, 566, 680, 793, 906, 974, 1042, 1133k $k_{B} T$; symbols are simulation data and lines serve as guides to the eye. The lowest reported value of $D$ is taken as the nonergodicity limit; (b) Phase diagram showing $\beta A$ as a function of $\phi$, extracted by taking the iso- $D$ lines from (a). The dashed line signals the onset of the glass region; state points with the same color coding have the same value of diffusion coefficient. 


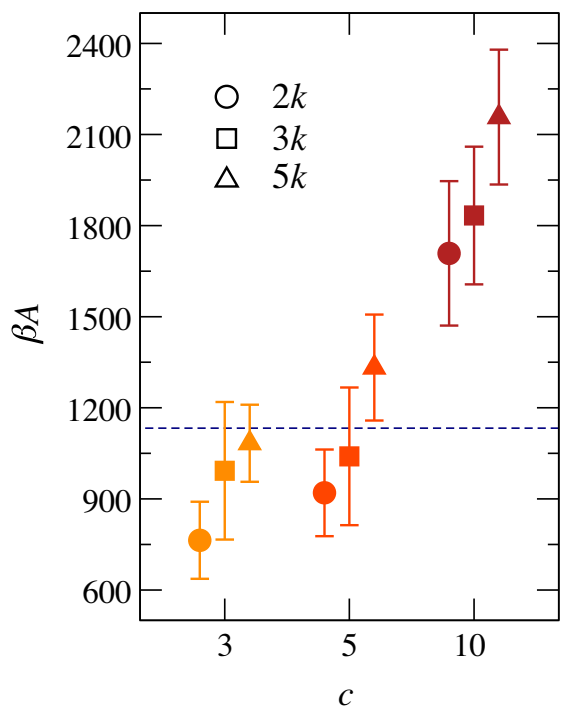

FIG. 5. Dependence of the 2D Hertzian strength $A$ on the crosslinker concentration for microgels of various sizes. Values of $\beta A$ are extracted via the theoretical calculation of $Y$ and $\sigma_{\text {ext }}$ for microgels assembled with $N \approx 2000$ (circles), 3000 (squares), and 5000 (triangles) monomers for $c=3 \%$ (orange), 5\% (red), and $10 \%$ (dark red). Symbols are slightly displaced on the $x$ axis to enhance readability. The dashed line indicates the approximate largest value of the Hertzian strength for which a reentrant transition could be observed (see Fig. 4). Data are averaged over four different topologies for each combination of $N$ and $c$.

Crucially, thanks to the knowledge of the functional form of the potential, we can now predict the experimental features of microgels that will most likely show a reentrant behavior. Indeed, since the repulsive Hertzian strength $A$ depends on Young's modulus and on the effective diameter of the particles, we need to consider microgels whose combined spreading and elastic properties at the interface fall into the predicted reentrant range. It turns out that we need to focus on microgels with relatively small size, since a reduction of the particle diameter strongly affects the value of the Hertzian strength, which depends quadratically on it. To be more precise, we perform additional simulations of microgels made of 2000 and 3000 monomers, besides those with approximately 5000 monomers. In order to avoid long computational times for the calculation of the effective interactions, we directly determine the Hertzian strengths via elasticity theory calculations and by measuring $\sigma_{\text {ext }}$ for single particles with different sizes and crosslinker concentrations at the interface.

We report the estimated repulsive strengths as a function of $c$ in Fig. 5 and find that soft and small microgels have an Hertzian strength that falls in the range where a reentrant behavior of the dynamics is present, according to the phase diagram in Fig. 4(b). We also confirm that the value of Young's modulus does not exhibit a strong size dependence, especially for $c=3 \%$ and $5 \%$ (see Supplemental Material [51]), in qualitative agreement with experimental findings on microgels of different sizes [85-87]. Hence, from this analysis, we conclude that highly cross-linked microgels will always display glassy dynamics at the interface, independent of their size. Instead, low-crosslinked microgels whose Young's modulus at the interface is around $0.1-0.3 k_{B} T / \sigma_{m}^{2}$ and whose extended size is between approximately 35 and $50 \sigma_{m}$ are expected to show a reentrant dynamics. Thanks to the mapping performed in previous comparisons with experiments $[29,41]$, we are now able to convert these predictions to real values which, for laboratory microgels, correspond to hydrodynamic diameters in bulk $\lesssim 200 \mathrm{~nm}$. This value is well within the commonly investigated experimental range and offers the additional advantage that capillary effects should be less relevant. Therefore, adsorbed microgels of small size and low-crosslinking ratio constitute a realistic model system to experimentally investigate the presence of a reentrant dynamics, long postulated in the realm of soft colloids.

It is also instructive to think where this regime can be observed in terms of compression isotherms to which experiments typically refer. From the present calculations, we estimate that the value of the area fraction is reduced by about a half as compared to the corona-corona contact at low densities. Even though these compressions are not too high [37], a number of critical issues may emerge, and these are ultimately linked to the real-time visualization of the microgels at the interface, which is essential to retrieve dynamical information from the interfacial microgel assembly and thus observe the reentrant melting at high densities. Currently, most of the studies are performed ex situ by means of Atomic Force Microscopy (AFM) on silica wafer or similar techniques, from which only structural static information can be extracted. However, the realtime visualization is just one of the options for the experimental verification since other approaches could be devised. For instance, one could imagine to put forward a rheological investigation and analyze the response of the microgel ensemble at different packing fractions. Overall, we believe that our predictions will stimulate experimental work to confirm the predicted dynamical behavior for microgels at interfaces.

\section{CONCLUSIONS AND PERSPECTIVES}

In summary, in this work we provide the first numerical estimate of the two-body effective interaction potential of microgel particles adsorbed at an interface. The complex arrangement of such particles on the interfacial plane is thus rationalized with a simple functional form that reveals that they interact like effective elastic disks with Young's modulus that increases with the cross-linker concentration. Notably, the values of the elastic moduli at the interface, after appropriate rescaling, are found to be roughly one order of magnitude higher than the one measured in bulk, as also confirmed by elasticity theory calculation of single microgel particles. This can be attributed to the dominant 
effects of the interfacial tension, which controls the response of the polymer network to an external stress, making it much stiffer and more resistant to deformation with respect to the same network in good solvent conditions. This result has profound consequences on the properties of a generic interfacial assembly of soft colloids, not limited to microgel particles. Indeed, we expect that the reduced mobility of the polymer chains and their enhanced stiffness should be taken into account in the development of novel materials that rely on deformable constituents of any kind. As demonstrated by our results, this effect should be expected at interfacial conditions with large surface tensions, independent of the presence of intrinsic charges in the material or in the fluids. In this respect, our results call for direct experimental verification which could unambiguously shed light on these aspects.

From a more fundamental perspective, we clearly demonstrate that the knowledge that is gained on the bulk properties of soft colloids cannot be directly transferred to the interface, which should be considered as a separate case, where particles behave and interact in a different way. Indeed, here we numerically show that microgels at interfaces follow the Hertzian predictions as 2D objects even at very short separations, well beyond the smalldeformation regime. This is in stark contrast to microgels in bulk, where the validity of the Hertzian model is found to apply only up to interactions of the order of few $k_{B} T$, corresponding to moderately large center-to-center distances and small deformations. This behavior in bulk is due to the internal morphology of the microgel that imposes multiple length scales to be included in the description of the collective behavior. Instead, at the interface, the behavior is fully dominated by the very extended coronas. We can thus state that microgels do have distinct properties depending on the environment in which they are placed, opening up new avenues for their exploitation. A similar scenario should be expected for any soft particle adsorbed at interfaces with respect to the corresponding behavior in bulk conditions.

The extensive analysis of the multiparticle dynamics further evidenced the emergence of reentrant dynamics, where the system behaves as an ergodic fluid up to very large densities, well above individual particles contact, sometimes loosely called jamming. Experimentally, small (nanosized) soft microgels appear to be the ideal candidates to verify our theoretical predictions, as indicated by the values of Young's modulus and of the interfacial extension at which the reentrance is observed. In addition, small colloids are the least likely to experience capillary attractions at the interface, and hence will behave more similarly to the ones we simulated.

It will be important in the future to extend this study to crowded configurations to investigate the validity of the present results at considerably high packing fractions where additional mechanisms, like faceting or interpenetration, may become relevant. Under these conditions, many-body effects should also play a prominent role. In our current treatment, we cannot quantify the influence of many-body interactions due to severe computational limitations, since a huge number of particles should be used. Our approach provides, in this respect, a first step toward a comprehensive description of microgel interactions at a microscopic level. Similar considerations should be extended to microgels in bulk conditions, for which high-density states still require appropriate theoretical assessment. The analysis can be further broadened to other microgel topologies that have recently gained considerable attention, such as hollow [88], ultra-low-cross-linked [28], or anisotropic ones [89].

All in all, our study opens the way for the investigation of microgels at the interface as a simple realization of 2D elastic particles. We expect that the evidence reported here will have important consequences on the study of twodimensional elastic objects at the fundamental level $[90,91]$ and for the clever design of composite materials [92-94].

\section{METHODS}

\section{A. Modeling and simulation details}

Microgels are assembled starting from an ensemble of two- and four-folded patchy particles in a spherical cavity. Bivalent and tetravalent particles mimic, respectively, $\mathrm{N}$-isopropylacrylamide monomers and N,N'-methylenebisacrylamide cross-linkers in a chemical synthesis [49]. Microgel assembly is performed with the oxDNA simulation package [95]. The topology of the polymer network, whose monomers have diameter $\sigma_{m}$ (which also sets the unit of length) is then fixed by means of a classical bead-spring model [47] that amounts to the Weeks-Chandler-Andersen (WCA) potential for nonbonded monomers, and a sum of the WCA and the finitely extensible nonlinear elastic (FENE) potentials for bonded ones:

$V_{\mathrm{WCA}}(r)= \begin{cases}4 \epsilon\left[\left(\frac{\sigma_{m}}{r}\right)^{12}-\left(\frac{\sigma_{m}}{r}\right)^{6}\right]+\epsilon & \text { if } r \leq 2^{\frac{1}{6}} \sigma_{m} \\ 0 & \text { otherwise }\end{cases}$

$V_{\mathrm{FENE}}(r)=-\epsilon k_{F} R_{0}^{2} \ln \left[1-\left(\frac{r}{R_{0} \sigma_{m}}\right)^{2}\right]$ if $r<R_{0} \sigma_{m}$

with $k_{F}=15$ a dimensionless spring constant and $R_{0}=$ 1.5 the maximum extension of the bond. The method ensures that each designed microgel is made by a disordered cross-linked polymer network with a core-corona structure as in real microgels. Furthermore, the adoption of a designing force acting on the cross-linkers during the network assembly improves considerably the agreement between the numerical and experimental form factors as described in Ref. [41]. Because of the fact that each microgel is assembled independently, the topology between 
different particles may slightly vary in terms of internal connectivity and density profiles, while maintaining the same polymer chains distribution and the same macroscopic features such as the presence of a core-corona structure or a density profile that slowly decrease toward the outer shells. We stress the importance to have realistic topologies that can closely match experimental data [29], since the inner structure of the microgel particle influence both the elastic properties and the effective interactions.

In the present work, we employ microgels with approximately 5000 monomers and three different molar crosslinker concentrations $c$, namely, 3\%, 5\%, and $10 \%$. The radius of the confining sphere into which microgels are assembled is set to $25 \sigma_{m}$. We also evaluate the elasticity of smaller microgels with approximately 2000 and 3000 particles assembled in the same way, and maintaining the same internal monomer density. The effective interactions are assessed for a second microgel topology for $c=5 \%$ (see Supplemental Material [51]), while the analysis of the elastic properties as a function of the microgel size are studied over four independent microgel topologies.

According to previous works [29,50], the solvent is modeled within the dissipative particle dynamics (DPD) framework [96]. The interactions are described by three forces, conservative $\vec{F}_{i j}^{C}$, dissipative $\vec{F}_{i j}^{D}$, and random $\vec{F}_{i j}^{R}$, of form

$$
\begin{gathered}
\vec{F}_{i j}^{C}= \begin{cases}a\left(1-r_{i j} / r_{c}\right) \hat{r}_{i j} & \text { if } r_{i j}<r_{c}, \\
0 & \text { otherwise, }\end{cases} \\
\vec{F}_{i j}^{D}=-\xi w^{D}\left(r_{i j}\right)\left(\hat{r}_{i j} \cdot \vec{v}_{i j}\right) \hat{r}_{i j}, \\
\vec{F}_{i j}^{R}=\sigma_{R} w^{R}\left(r_{i j}\right) \theta_{i j}(\Delta t)^{-1 / 2} \hat{r}_{i j},
\end{gathered}
$$

where $\vec{r}_{i j}=\vec{r}_{i}-\vec{r}_{j}$, with $\vec{r}_{i}$ the position of particle $i$, $r_{i j}=\left|\vec{r}_{i j}\right|, \hat{r}_{i j}=\vec{r}_{i j} / r_{i j}, r_{c}$ the cutoff radius, $\vec{v}_{i j}=\vec{v}_{i}-$ $\vec{v}_{j}$ with $\vec{v}_{i}$ the velocity of particle $i, a$ is the maximum repulsion between two particles, $\theta_{i j}$ is a Gaussian random number with zero mean and unit variance, and $\xi$ is the friction coefficient [96]. To ensure that Boltzmann equilibrium is reached, $w^{D}\left(r_{i j}\right)=\left[w^{R}\left(r_{i j}\right)\right]^{2}$ and $\sigma_{R}^{2}=2 \xi k_{B} T$ with $k_{B}$ the Boltzmann constant and $T$ the temperature.

In order to reproduce a water-hexane $(w-h)$ interface, we choose $a_{w w}=a_{h h}=8.8, a_{h w}=31.1$. While in principle it is possible to change these parameters in order to obtain a different surface tension, no significant difference is expected in the microgel-microgel interaction potential, given that the distribution of microgel monomers remains unaltered [29]. Instead, for the monomer-solvent interactions, we choose $a_{m w}=4.5$ and $a_{m h}=5.0$. The cutoff radius is always set to be $r_{c}=1.9 \sigma_{m}$ and the reduced solvent density $\rho_{\mathrm{DPD}}=4.5$ [29]. Depending on the microgel size, up to approximately 750000 solvent particles are inserted in the simulation box. To analyze the elastic properties of microgels in bulk, we also run bulk simulations with explicit solvent. In there, the solvent-solvent parameters are not varied with respect to interfacial simulations, while $a_{m s}=1.0$, ensuring good solvent conditions. A more detailed discussion on how these parameters are determined can be found in Ref. [29].

Simulations are carried out using the LAMMPS simulation package [97]. The equations of motion are integrated with a velocity-Verlet algorithm. The reduced temperature $T^{*}=$ $k_{B} T / \epsilon$ is always set to 1.0 via the DPD thermostat [50]. Length, mass, and energy are given in units of $\sigma_{m}, m, \epsilon$, respectively. DPD repulsion parameters $a$ are in units of $\epsilon / \sigma_{m}$.

The phase behavior of the 2D Hertzian potential is assessed by means of molecular dynamics simulations in two dimensions with 5000 particles of unit mass $m$ and diameter $\sigma_{\text {eff }}$, interacting via Eq. (1). We use $\sigma_{\text {eff }}$ as the unit of length, so that the area fraction is defined as $\phi=(\pi / 4)\left\langle\sigma_{\text {eff }}^{2}\right\rangle \rho$, with $\rho$ the number density. We fix $k_{B} T=1$, which defines the unit of energy, via a Langevin thermostat; time is in units $\sqrt{m \sigma_{\text {eff }}^{2} / k_{B} T}$. To avoid crystallization, we set the polydispersity to $p=0.2$. We analyze a range of $\phi$ from 0.8 to 2.8 for a Hertzian strength defined as $A=\pi Y \sigma_{\text {eff }}^{2} /(2 \ln 2)$, that goes from 220 to $1150 k_{B} T$. We note that $A$ has units of energy over length squared, meaning that it changes value depending on the units of measurement used ( $\sigma_{m}$ and $\sigma_{\text {eff }}$ for the monomerresolved system and the coarse-grained systems, respectively). For all $\phi$ and $A$, we monitor the presence of a liquidlike disordered structure by calculating the radial distribution function (see Supplemental Material [51]).

To determine the glass region in the $2 \mathrm{D}$ phase diagram, we run simulations for approximately $2 \times 10^{7}$ time steps, and we calculate the mean-squared displacement $\left\langle\Delta r^{2}\right\rangle$ of the particles, extracting the long-time self-diffusion coefficient $D$ :

$$
D=\lim _{t \rightarrow \infty} \frac{\left\langle\Delta r^{2}\right\rangle}{4 t},
$$

where $t$ is the simulation time. Since we are interested only in providing a state diagram assessment, we do not perform an extensive characterization of the glassy dynamics of the system, and we just monitor the onset of nonergodicity within the timescale of our simulations [46]. We attribute this condition to state points where we find $D \lesssim 2.5 \times 10^{-5}$, roughly 3 orders of magnitude lower than the corresponding low-density value. Under these conditions, the system has become so slow that aging is present within our simulation time window.

\section{B. Calculation of the effective interaction potential}

The two-body effective potential between the microgels at the interface is evaluated by means of the umbrella 
sampling technique in explicit solvent [52-54]. This method allows us to uniformly sample all distances between the centers of mass of the microgels by adding a harmonic potential between them. For each sampled window $i$, we evaluate the probability distribution $P\left(r, \Delta_{i}\right)$ of finding the microgels' centers of mass at distance $r$ given the equilibrium length of the spring $\Delta_{i}$. The final probability for the entire range of explored distances is obtained by first removing the contribution of the bias potential and by subsequently merging $P\left(r, \Delta_{i}\right)$ into $P(r)$ for all the windows via a least-squares method. Finally, the potential of mean force $V_{\text {eff }}$ is retrieved knowing that

$$
V_{\text {eff }}=-k_{B} T \ln [P(r)]+C,
$$

where $C$ is such that $V_{\text {eff }}(r \rightarrow \infty)=0$. The major drawback of studying the effective interactions with an explicit solvent model is the computational cost required to carry out the simulations: About two months on about $80 \mathrm{CPU}$ cores are needed to investigate a range from 20 to $30 \Delta_{i}$.

\section{Assessment of the elastic moduli}

Following Ref. [98], the elastic energy $U$ of a twodimensional object can be written as a function of the invariants $J$ and $I$ of the strain tensor as

$$
U(J, I)=U_{0}+W(J)+W(I),
$$

where $U_{0}$ is the energy of a reference configuration that is taken as the average ellipse adopted by equilibrium configurations of the microgels at the interface. Its semiaxes $s_{1}$ and $s_{2}$ are obtained by the gyration tensor built via the two-dimensional convex hull on the plane of the interface. We approximate $W$ with the corresponding potentials of mean force

$$
W(X)=-k_{B} T \ln P(X)+D
$$

with $X=J, I . P(X)$ is the respective probability distribution and $D$ an arbitrary constant. These potentials can then be fitted to appropriate functions

$$
f\left(X ; M_{X}, X_{0}, \gamma, C\right)=M_{X}\left(X-X_{0}\right)^{\gamma}+C
$$

with $\gamma=2$ when $X=J$ and $\gamma=1$ when $X=I$, to obtain $M_{J}$ and $M_{I}$. In the Supplemental Material [51], we report, as an example for $c=10 \%$, the simulation outcomes and their relative fits both for the microgel at the interface and in bulk. The elastic moduli are then readily obtained as

$$
\begin{gathered}
K=\frac{2 M_{J}}{S}, \\
G=\frac{2 M_{I}}{S}
\end{gathered}
$$

with $S=\pi s_{1} s_{2} . Y$ and $\nu$ depend only on $K$ and $G$ as [67]

$$
\begin{aligned}
& \nu=\frac{K-G}{K+G}, \\
& Y=\frac{4 K G}{K+G} .
\end{aligned}
$$

Similar expressions can be derived for the 3D case and can be found, for instance, in Ref. [42] (see also below).

The particular choice of $W$ as a function of $J$ and $I$ depends on the specific elastic model employed. Here, we consider the Mooney-Rivlin model for which the elastic energy reads $[60,61]$

$$
U(J, I)=U_{0}+\frac{S}{2}\left[K(J-1)^{2}+G(I-2)\right] .
$$

We further check that the obtained results do not crucially depend on the specific form of $W$. To this aim, we also employ the linear elastic model (Hookean) [60,99] and the Saint-Venant-Kirchhoff model [100,101], finding results for the moduli, particularly Young's modulus, that are very close to the ones we present in the main text. They display the same increase with respect to the bulk model and a similar monotonic increase with $c$.

To convert the 2D moduli into 3D ones, we consider that for two-dimensional objects the stress normal to the interfacial plane is zero. Under these conditions, there exist relations to convert $2 \mathrm{D}$ moduli into $3 \mathrm{D}$ ones by assuming that the 2D object has a given (small) thickness $h$ :

$$
\begin{gathered}
G^{(3)}=G^{(2)} / h, \\
Y^{(3)}=Y^{(2)} / h, \\
K^{(3)}=\frac{4 G^{(2)} K^{(2)}}{3 h\left(3 G^{(2)}-K^{(2)}\right)},
\end{gathered}
$$

where $X^{(3)}$ indicates the converted 3D moduli (in units of $k_{B} T / \sigma_{m}^{3}$ ) from the 2D results $X^{(2)}$ (in units of $k_{B} T / \sigma_{m}^{2}$ ) with $X=G, Y, K$. Also, we have that [62]

$$
\nu^{(3)}=\nu^{(2)} .
$$

In our case, we consider $h$ to be roughly equal to the monomer size $\sigma_{m}$, as in the outer shells chains do not pile up, but remain confined to the interfacial plane, providing the dominant contribution to the elastic response of the microgels. Furthermore, as reported in Ref. [29] based on AFM studies, the realistic width of a microgel corona is below $7 \mathrm{~nm}$, that is a fully compatible size to the one we extract for a single in silico microgel monomer by comparing the form factors of numerical and laboratory microgels [41]. 


\section{ACKNOWLEDGMENTS}

We thank M. A. Fernández-Rodríguez, F. Grillo, L. Isa, J. Vialetto, and P. Ziherl for valuable discussions. We acknowledge financial support from the European Research Council (ERC Consolidator Grant No. 681597, MIMIC), from the European Union's Horizon 2020 research and innovation program (Grant No. 731019, EUSMI), from MIUR (FARE Project No. R16XLE2X3L, SOFTART), and from Regione Lazio, through L. R. 13/08 (Progetto Gruppo di Ricerca GELARTE, n.prot.85-2017-15290). The authors gratefully acknowledge the computing time granted by EUSMI on the supercomputer JURECA at the Jülich Supercomputing Centre (JSC), and by CINECA under the ISCRA initiative.

[1] V. Lubchenko and P. G. Wolynes, Theory of Structural Glasses and Supercooled Liquids, Annu. Rev. Phys. Chem. 58, 235 (2007).

[2] S. Torquato, Perspective: Basic Understanding of Condensed Phases of Matter via Packing Models, J. Chem. Phys. 149, 020901 (2018).

[3] L. A. Lyon and A. Fernandez-Nieves, The Polymer/ Colloid Duality of Microgel Suspensions, Annu. Rev. Phys. Chem. 63, 25 (2012).

[4] M. Karg, A. Pich, T. Hellweg, T. Hoare, L. A. Lyon, J. J. Crassous, D. Suzuki, R. A. Gumerov, S. Schneider, and I. I. Potemkin, Nanogels and Microgels: From Model Colloids to Applications, Recent Developments, and Future Trends, Langmuir 35, 6231 (2019).

[5] A. Martín-Molina and M. Quesada-Pérez, A Review of Coarse-Grained Simulations of Nanogel and Microgel Particles, J. Mol. Liq. 280, 374 (2019).

[6] J. Oberdisse and T. Hellweg, Recent Advances in StimuliResponsive Core-Shell Microgel Particles: Synthesis, Characterisation, and Applications, Colloid Polym. Sci. (2020).

[7] M. Rey, M. A. Fernandez-Rodriguez, M. Karg, L. Isa, and N. Vogel, Poly-N-isopropylacrylamide Nanogels and Microgels at Fluid Interfaces, Acc. Chem. Res. 53, 414 (2020).

[8] R. Pelton, Temperature-Sensitive Aqueous Microgels, Adv. Colloid Interface Sci. 85, 1 (2000).

[9] E. Siemes, O. Nevskyi, D. Sysoiev, S. K. Turnhoff, A. Oppermann, T. Huhn, W. Richtering, and D. Wöll, Nanoscopic Visualization of Cross-Linking Density in Polymer Networks with Diarylethene Photoswitches, Angew. Chem., Int. Ed. Engl. 57, 12280 (2018).

[10] M. J. Bergman, N. Gnan, M. Obiols-Rabasa, J.-M. Meijer, L. Rovigatti, E. Zaccarelli, and P. Schurtenberger, A New Look at Effective Interactions between Microgel Particles, Nat. Commun. 9, 5039 (2018).

[11] F. Scheffold, P. Díaz-Leyva, M. Reufer, N. B. Braham, I. Lynch, and J. L. Harden, Brushlike Interactions between Thermoresponsive Microgel Particles, Phys. Rev. Lett. 104, 128304 (2010).

[12] G. M. Conley, C. Zhang, P. Aebischer, J. L. Harden, and F. Scheffold, Relationship between Rheology and Structure of Interpenetrating, Deforming and Compressing Microgels, Nat. Commun. 10, 2436 (2019).

[13] M.-h. Kwok, G. Sun, and T. Ngai, Microgel Particles at Interfaces: Phenomena, Principles, and Opportunities in Food Sciences, Langmuir 35, 4205 (2019).

[14] M. Wang, A. M. Mihut, E. Rieloff, A. P. Dabkowska, L. K. Månsson, J. N. Immink, E. Sparr, and J. J. Crassous, Assembling Responsive Microgels at Responsive Lipid Membranes, Proc. Natl. Acad. Sci. U.S.A. 116, 5442 (2019).

[15] M. C. Tatry, E. Laurichesse, A. Perro, V. Ravaine, and V. Schmitt, Kinetics of Spontaneous Microgels Adsorption and Stabilization of Emulsions Produced Using Microfluidics, J. Colloid Interface Sci. 548, 1 (2019).

[16] W. Richtering, Responsive Emulsions Stabilized by Stimuli-Sensitive Microgels: Emulsions with Special Non-Pickering Properties, Langmuir 28, 17218 (2012).

[17] H. Monteillet, M. Workamp, J. Appel, J. M. Kleijn, F. A. Leermakers, and J. Sprakel, Ultrastrong Anchoring yet Barrier-Free Adsorption of Composite Microgels at Liquid Interfaces, Adv. Mater. Interfaces 1, 1300121 (2014).

[18] M. Rey, M. Á. Fernández-Rodríguez, M. Steinacher, L. Scheidegger, K. Geisel, W. Richtering, T. M. Squires, and L. Isa, Isostructural Solid-Solid Phase Transition in Monolayers of Soft Core-Shell Particles at Fluid Interfaces: Structure and Mechanics, Soft Matter 12, 3545 (2016).

[19] T. Dotera, T. Oshiro, and P. Ziherl, Mosaic Two-Lengthscale Quasicrystals, Nature (London) 506, 208 (2014).

[20] M. Zu, P. Tan, and N. Xu, Forming Quasicrystals by Monodisperse Soft Core Particles, Nat. Commun. 8, 2089 (2017).

[21] S. Schmidt, T. Liu, S. Rütten, K.-H. Phan, M. Möller, and W. Richtering, Influence of Microgel Architecture and Oil Polarity on Stabilization of Emulsions by Stimuli-Sensitive Core-Shell Poly (n-isopropylacrylamide-co-methacrylic Acid) Microgels: Mickering versus Pickering Behavior?, Langmuir 27, 9801 (2011).

[22] T. Liu, S. Seiffert, J. Thiele, A. R. Abate, D. A. Weitz, and W. Richtering, Non-Coalescence of Oppositely Charged Droplets in $\mathrm{pH}$-Sensitive Emulsions, Proc. Natl. Acad. Sci. U.S.A. 109, 384 (2012).

[23] F. Grillo, M.-A. Fernandez-Rodriguez, M.-N. Antonopoulou, D. Gerber, and L. Isa, Self-Templating Assembly of Soft Microparticles into Complex Tessellations, arXiv: 1911.13171.

[24] K. Volk, F. Deißenbeck, S. Mandal, H. Löwen, and M. Karg, Moiré and Honeycomb Lattices through SelfAssembly of Hard-Core/Soft-Shell Microgels: Experiment and Simulation, Phys. Chem. Chem. Phys. 21, 19153 (2019).

[25] M. Á. Fernández-Rodríguez, R. Elnathan, R. Ditcovski, F. Grillo, G. M. Conley, F. Timpu, A. Rauh, K. Geisel, T. Ellenbogen, and R. Grange, Tunable 2D Binary Colloidal Alloys for Soft Nanotemplating, Nanoscale 10, 22189 (2018).

[26] B. M. Rey, R. Elnathan, R. Ditcovski, K. Geisel, M. Zanini, M. Á. Fernández-Rodríguez, V. V. Naik, A. Frutiger, W. Richtering, and T. Ellenbogen, Fully Tunable 
Silicon Nanowire Arrays Fabricated by Soft Nanoparticle Templating, Nano Lett. 16, 157 (2016).

[27] K. Geisel, L. Isa, and W. Richtering, Unraveling the 3D Localization and Deformation of Responsive Microgels at Oil/Water Interfaces: A Step Forward in Understanding Soft Emulsion Stabilizers, Langmuir 28, 15770 (2012).

[28] A. Scotti, S. Bochenek, M. Brugnoni, M.-A. FernandezRodriguez, M. F. Schulte, J. Houston, A. P. Gelissen, I. I. Potemkin, L. Isa, and W. Richtering, Exploring the Colloid-to-Polymer Transition for Ultra-Low Crosslinked Microgels from Three to Two Dimensions, Nat. Commun. 10, 1418 (2019).

[29] F. Camerin, M. A. Fernandez-Rodriguez, L. Rovigatti, M.-N. Antonopoulou, N. Gnan, A. Ninarello, L. Isa, and E. Zaccarelli, Microgels Adsorbed at Liquid-Liquid Interfaces: A Joint Numerical and Experimental Study, ACS Nano 13, 4548 (2019).

[30] J. Maldonado-Valderrama, T. del Castillo-Santaella, I. Adroher-Benítez, A. Moncho-Jordá, and A. MartínMolina, Thermoresponsive Microgels at the Air-Water Interface: The Impact of the Swelling State on Interfacial Conformation, Soft Matter 13, 230 (2017).

[31] G. Agrawal and R. Agrawal, Stimuli-Responsive Microgels and Microgel-Based Systems: Advances in the Exploitation of Microgel Colloidal Properties and Their Interfacial Activity, Polymers 10, 418 (2018).

[32] J. Harrer, M. Rey, S. Ciarella, H. Lowen, L. M. Janssen, and N. Vogel, Stimuli-Responsive Behavior of PNiPAm Microgels under Interfacial Confinement, Langmuir 35, 10512 (2019).

[33] D. J. Arismendi-Arrieta and A. J. Moreno, Deformability and Solvent Penetration in Soft Nanoparticles at LiquidLiquid Interfaces, J. Colloid Interface Sci. 570, 212 (2020).

[34] O.S. Deshmukh, A. Maestro, M. H. Duits, D. van den Ende, M. C. Stuart, and F. Mugele, Equation of State and Adsorption Dynamics of Soft Microgel Particles at an Air-Water Interface, Soft Matter 10, 7045 (2014).

[35] S. Bochenek, A. Scotti, W. Ogieglo, M. A. FernandezRodriguez, M. F. Schulte, R. A. Gumerov, N. V. Bushuev, I. I. Potemkin, M. Wessling, L. Isa et al., Effect of the $3 D$ Swelling of Microgels on Their 2D Phase Behavior at the Liquid-Liquid Interface, Langmuir 35, 16780 (2019).

[36] C. Picard, P. Garrigue, M.-C. Tatry, V. Lapeyre, S. Ravaine, V. Schmitt, and V. Ravaine, Organization of Microgels at the Air-Water Interface under Compression: Role of Electrostatics and Cross-Linking Density, Langmuir 33, 7968 (2017).

[37] L. Scheidegger, M. Á. Fernández-Rodríguez, K. Geisel, M. Zanini, R. Elnathan, W. Richtering, and L. Isa, Compression and Deposition of Microgel Monolayers from Fluid Interfaces: Particle Size Effects on Interface Microstructure and Nanolithography, Phys. Chem. Chem. Phys. 19, 8671 (2017).

[38] M. Rey, A. D. Law, D. M. A. Buzza, and N. Vogel, Anisotropic Self-Assembly from Isotropic Colloidal Building Blocks, J. Am. Chem. Soc. 139, 17464 (2017).

[39] A. Maestro, Tailoring the Interfacial Assembly of Colloidal Particles by Engineering the Mechanical Properties of the Interface, Curr. Opin. Colloid Interface Sci. 39, 232 (2019).
[40] O. S. Deshmukh, D. van den Ende, M. C. Stuart, F. Mugele, and M. H. Duits, Hard and Soft Colloids at Fluid Interfaces: Adsorption, Interactions, Assembly \& Rheology, Adv. Colloid Interface Sci. 222, 215 (2015).

[41] A. Ninarello, J. J. Crassous, D. Paloli, F. Camerin, N. Gnan, L. Rovigatti, P. Schurtenberger, and E. Zaccarelli, Modeling Microgels with a Controlled Structure across the Volume Phase Transition, Macromolecules 52, 7584 (2019).

[42] L. Rovigatti, N. Gnan, A. Ninarello, and E. Zaccarelli, Connecting Elasticity and Effective Interactions of Neutral Microgels: The Validity of the Hertzian Model, Macromolecules 52, 4895 (2019).

[43] D. Vlassopoulos and M. Cloitre, Tunable Rheology of Dense Soft Deformable Colloids, Curr. Opin. Colloid Interface Sci. 19, 561 (2014).

[44] R. A. LaCour, C. S. Adorf, J. Dshemuchadse, and S. C. Glotzer, The Influence of Softness on the Stability of Binary Colloidal Crystals, ACS Nano 13, 13829 (2019).

[45] N. Gnan and E. Zaccarelli, The Microscopic Role of Deformation in the Dynamics of Soft Colloids, Nat. Phys. 15, 683 (2019).

[46] L. Berthier, A. J. Moreno, and G. Szamel, Increasing the Density Melts Ultrasoft Colloidal Glasses, Phys. Rev. E 82, 060501(R) (2010).

[47] K. Kremer and G. S. Grest, Dynamics of Entangled Linear Polymer Melts: A Molecular-Dynamics Simulation, J. Chem. Phys. 92, 5057 (1990).

[48] G. S. Grest and K. Kremer, Molecular Dynamics Simulation for Polymers in the Presence of a Heat Bath, Phys. Rev. A 33, 3628 (1986).

[49] N. Gnan, L. Rovigatti, M. Bergman, and E. Zaccarelli, In Silico Synthesis of Microgel Particles, Macromolecules 50, 8777 (2017).

[50] F. Camerin, N. Gnan, L. Rovigatti, and E. Zaccarelli, Modelling Realistic Microgels in an Explicit Solvent, Sci. Rep. 8, 14426 (2018).

[51] See Supplemental Material at http://link.aps.org/ supplemental/10.1103/PhysRevX.10.031012 for further considerations on the microgel-microgel effective interaction potential, on the calculation of the elastic moduli, and on the multiparticle dynamical response.

[52] R. Blaak, B. Capone, C. N. Likos, and L. Rovigatti, Accurate Coarse-Grained Potentials for Soft Matter Systems, in Computational Trends in Solvation and Transport in Liquids-Lecture Notes, edited by G. Sutmann, J. Grotendorst, G. Gompper, and D. Marx (Forschungszentrum Jülich, IAS Series, 2015), Vol. 28.

[53] C. N. Likos, Effective Interactions in Soft Condensed Matter Physics, Phys. Rep. 348, 267 (2001).

[54] N. Gnan, E. Zaccarelli, and F. Sciortino, Casimir-like Forces at the Percolation Transition, Nat. Commun. 5, 3267 (2014).

[55] F. Gerl and A. Zippelius, Coefficient of Restitution for Elastic Disks, Phys. Rev. E 59, 2361 (1999).

[56] H. Hayakawa and H. Kuninaka, Simulation and Theory of the Impact of Two-Dimensional Elastic Disks, Chem. Eng. Sci. 57, 239 (2002).

[57] M. Rey, X. Hou, J. S. J. Tang, and N. Vogel, Interfacial Arrangement and Phase Transitions of PNiPAm Microgels 
with Different Crosslinking Densities, Soft Matter 13, 8717 (2017).

[58] S. Huang, K. Gawlitza, R. von Klitzing, W. Steffen, and G. K. Auernhammer, Structure and Rheology of Microgel Monolayers at the Water/Oil Interface, Macromolecules 50, 3680 (2017).

[59] A. El-Tawargy, D. Stock, M. Gallei, W. Ramadan, M. Shams El-Din, G. Reiter, and R. Reiter, Multiple Structural Transitions in Langmuir Monolayers of Charged Soft-Shell Nanoparticles, Langmuir 34, 3909 (2018).

[60] I. Doghri, Mechanics of Deformable Solids: Linear, Nonlinear, Analytical and Computational Aspects (Springer Science \& Business Media, New York, 2013).

[61] A. Aggarwal, E. R. May, C. L. Brooks III, and W. S. Klug, Nonuniform Elastic Properties of Macromolecules and Effect of Prestrain on Their Continuum Nature, Phys. Rev. E 93, 012417 (2016).

[62] S. Torquato, Random Heterogeneous Materials Microstructure and Macroscopic Properties (Springer, New York, 2002).

[63] M. A. Meyers and K. K. Chawla, Mechanical Behavior of Materials (Cambridge University Press, Cambridge, England, 2008).

[64] J. Hegemann, H.-H. Boltz, and J. Kierfeld, Elastic Capsules at Liquid-Liquid Interfaces, Soft Matter 14, 5665 (2018).

[65] T.-S. Lin, R. Wang, J. A. Johnson, and B. D. Olsen, Revisiting the Elasticity Theory for Real Gaussian Phantom Networks, Macromolecules 52, 1685 (2019).

[66] A. Kloczkowski, B. Erman, and J. Mark, Effect of NonGaussian Chains on Fluctuations of Junctions in Bimodal Networks, Polymer 43, 2569 (2002).

[67] S. Meille and E. J. Garboczi, Linear Elastic Properties of $2 D$ and 3D Models of Porous Materials Made from Elongated Objects, Model. Simul. Mater. Sci. Eng. 9, 371 (2001).

[68] S. Wellert, Y. Hertle, M. Richter, M. Medebach, D. Magerl, W. Wang, B. Deme, A. Radulescu, P. Muller-Buschbaum, and T. Hellweg, Inner Structure of Adsorbed Ionic Microgel Particles, Langmuir 30, 7168 (2014).

[69] T. Kyrey, J. Witte, V. Pipich, A. Feoktystov, A. Koutsioubas, E. Vezhlev, H. Frielinghaus, R. von Klitzing, S. Wellert, and O. Holderer, Influence of the Cross-Linker Content on Adsorbed Functionalised Microgel Coatings, Polymer 169, 29 (2019).

[70] M. F. Schulte, A. Scotti, A. P. Gelissen, W. Richtering, and A. Mourran, Probing the Internal Heterogeneity of Responsive Adsorbed to an Interface by a Sharp SFM Tip: Comparing Core-Shell and Hollow Microgels, Langmuir 34, 4150 (2018).

[71] J. C. Pàmies, A. Cacciuto, and D. Frenkel, Phase Diagram of Hertzian Spheres, J. Chem. Phys. 131, 044514 (2009).

[72] W. L. Miller and A. Cacciuto, Two-Dimensional Packing of Soft Particles and the Soft Generalized Thomson Problem, Soft Matter 7, 7552 (2011).

[73] Y. D. Fomin, E. Gaiduk, E. Tsiok, and V. Ryzhov, The Phase Diagram and Melting Scenarios of TwoDimensional Hertzian Spheres, Mol. Phys. 116, 3258 (2018).
[74] A. Scotti, A. R. Denton, M. Brugnoni, J. E. Houston, R. Schweins, I. I. Potemkin, and W. Richtering, Deswelling of Microgels in Crowded Suspensions Depends on Cross-Link Density and Architecture, Macromolecules 52, 3995 (2019).

[75] I. B. De Aguiar, T. Van de Laar, M. Meireles, A. Bouchoux, J. Sprakel, and K. Schroën, Deswelling and Deformation of Microgels in Concentrated Packings, Sci. Rep. 7, 10223 (2017).

[76] M. Urich and A. R. Denton, Swelling, Structure, and Phase Stability of Compressible Microgels, Soft Matter 12, 9086 (2016).

[77] E. Zaccarelli, G. Foffi, K. A. Dawson, S. V. Buldyrev, F. Sciortino, and P. Tartaglia, Confirmation of Anomalous Dynamical Arrest in Attractive Colloids: A Molecular Dynamics Study, Phys. Rev. E 66, 041402 (2002).

[78] G. Foffi, F. Sciortino, P. Tartaglia, E. Zaccarelli, F. Lo Verso, L. Reatto, K. A. Dawson, and C. N. Likos, Structural Arrest in Dense Star-Polymer Solutions, Phys. Rev. Lett. 90, 238301 (2003).

[79] N. Gnan, G. Das, M. Sperl, F. Sciortino, and E. Zaccarelli, Multiple Glass Singularities and Isodynamics in a CoreSoftened Model for Glass-Forming Systems, Phys. Rev. Lett. 113, 258302 (2014).

[80] F. L. Verso, J. A. Pomposo, J. Colmenero, and A. J. Moreno, Tunable Slow Dynamics in a New Class of Soft Colloids, Soft Matter 12, 9039 (2016).

[81] W. P. Krekelberg, T. Kumar, J. Mittal, J. R. Errington, and T. M. Truskett, Anomalous Structure and Dynamics of the Gaussian-Core Fluid, Phys. Rev. E 79, 031203 (2009).

[82] A.-M. Philippe, D. Truzzolillo, J. Galvan-Myoshi, P. Dieudonné-George, V. Trappe, L. Berthier, and L. Cipelletti, Glass Transition of Soft Colloids, Phys. Rev. E 97, 040601 (R) (2018).

[83] S. Gupta, J. Stellbrink, E. Zaccarelli, C. N. Likos, M. Camargo, P. Holmqvist, J. Allgaier, L. Willner, and D. Richter, Validity of the Stokes-Einstein Relation in Soft Colloids up to the Glass Transition, Phys. Rev. Lett. 115, 128302 (2015).

[84] A. Scotti, M. Brugnoni, C. G. Lopez, S. Bochenek, J. J. Crassous, and W. Richtering, Flow Properties Reveal the Particle-to-Polymer Transition of Ultra-Low Crosslinked Microgels, Soft Matter 16, 668 (2020).

[85] S. M. Hashmi and E. R. Dufresne, Mechanical Properties of Individual Microgel Particles through the Deswelling Transition, Soft Matter 5, 3682 (2009).

[86] P. Voudouris, D. Florea, P. van der Schoot, and H. M. Wyss, Micromechanics of Temperature Sensitive Microgels: Dip in the Poisson Ratio near the LCST, Soft Matter 9, 7158 (2013).

[87] F. Di Lorenzo, J. Hellwig, R. von Klitzing, and S. Seiffert, Macroscopic and Microscopic Elasticity of Heterogeneous Polymer Gels, ACS Macro Lett. 4, 698 (2015).

[88] K. Geisel, A. A. Rudov, I. I. Potemkin, and W. Richtering, Hollow and Core-Shell Microgels at Oil-Water Interfaces: Spreading of Soft Particles Reduces the Compressibility of the Monolayer, Langmuir 31, 13145 (2015).

[89] A. C. Nickel, A. Scotti, J. E. Houston, T. Ito, J. Crassous, J.S. Pedersen, and W. Richtering, Anisotropic Hollow Microgels That Can Adapt Their Size, Shape, and Softness, Nano Lett. 19, 8161 (2019). 
[90] Y. Peng, W. Li, F. Wang, T. Still, A. G. Yodh, and Y. Han, Diffusive and Martensitic Nucleation Kinetics in SolidSolid Transitions of Colloidal Crystals, Nat. Commun. 8, 14978 (2017).

[91] J. Vialetto, M. Anyfantakis, S. Rudiuk, M. Morel, and D. Baigl, Photoswitchable Dissipative Two-Dimensional Colloidal Crystals, Angew. Chem., Int. Ed. Engl. 131, 9243 (2019).

[92] E. Maza, C. von Bilderling, M. L. Cortez, G. Díaz, M. Bianchi, L. I. Pietrasanta, J. M. Giussi, and O. Azzaroni, Layer-by-Layer Assembled Microgels Can Combine Conflicting Properties: Switchable Stiffness and Wettability without Affecting Permeability, Langmuir 34, 3711 (2018).

[93] M. J. Serpe, C. D. Jones, and L. A. Lyon, Layer-by-Layer Deposition of Thermoresponsive Microgel Thin Films, Langmuir 19, 8759 (2003).

[94] B. S. Murray, Microgels at Fluid-Fluid Interfaces for Food and Drinks, Adv. Colloid Interface Sci. 271, 101990 (2019).
[95] L. Rovigatti, P. Sulc, I. Z. Reguly, and F. Romano, A Comparison between Parallelization Approaches in Molecular Dynamics Simulations on GPUs, J. Comput. Chem. 36, 1 (2015).

[96] R. D. Groot and P. B. Warren, Dissipative Particle Dynamics: Bridging the Gap between Atomistic and Mesoscopic Simulation, J. Chem. Phys. 107, 4423 (1997).

[97] S. Plimpton, Fast Parallel Algorithms for Short-Range Molecular Dynamics, J. Comput. Phys. 117, 1 (1995).

[98] L. R. G. Treloar, The Mechanics of Rubber Elasticity, Proc. R. Soc. A 351, 301 (1976).

[99] M. H. Sadd, Elasticity: Theory, Applications, and $\mathrm{Nu}$ merics (Academic Press, New York, 2009).

[100] L. Athanasopoulou and P. Ziherl, Phase Diagram of Elastic Spheres, Soft Matter 13, 1463 (2017).

[101] J. Riest, L. Athanasopoulou, S. A. Egorov, C. N. Likos, and P. Ziherl, Elasticity of Polymeric Nanocolloidal Particles, Sci. Rep. 5, 15854 (2015). 